\title{
Docencia en Urología Laparoscópica. Problemas. Soluciones
}

\author{
S. Novas Castro, M. Ruibal Moldes, M. González Martín \\ Servicio de Urología. Hospital Juan Canalejo. La Coruña.
}

Actas Urol Esp 2005: 29 (3): 241-243

\begin{abstract}
$\mathrm{A}_{\mathrm{s}}^{\mathrm{u}}$ unque la historia de la cirugía laparoscópica se remonta a principios del siglo XX, los comienzos recientes de la laparoscopia urológica datan del 1976 en el que Cortessi describió por primera vez la exploración de un paciente con un testículo no palpable mediante una laparoscopia. Tras estos primeros años que estuvieron marcados básicamente por una laparoscopia diagnóstica aparecieron los trabajos de Schuessler (1989) en la linfedenectomía pelviana en cáncer de próstata, las primeras nefrectomías realizadas por Clayman (1990) y la prostatectomía radical (Schuessler 1992) modificada por Gastón y Guillonneau en 1997 que hicieron que los urólogos volvieran a interesarse por ella desarrollándose de una manera importante en Europa especialmente en Francia, Bélgica y Alemania.

Es evidente que para su desarrollo ha habido muchas dificultades. Pero esto parece una cosa inherente al inicio de cualquier técnica que va a remover los cimientos de una especialidad. Recordemos los problemas que hubo en los años 70 para el desarrollo de la resección endoscópica y los trasplantes. Nadie podía imaginar hace 40 años el futuro de los trasplantes de órganos. Actualmente ya nadie pone en duda estas cuestiones.

En el caso de la cirugía laparoscópica la lista de los problemas para su desarrollo ha sido múltiple. Por un lado, hasta ahora hemos tenido la cirugia abierta que ha desarrollado técnicas muy estandarizadas, reproductibles, fáciles de enseñar y controlar. Sin embargo, las técnicas laparoscópicas hasta hace muy poco eran bastante aleatorias, no estaban estandarizadas, su desarrollo tecnológico era escaso, se aumentaba la duración operatoria, las complicaciones y eran difíciles de enseñar.

Ha sido un verdadero reto que ciertos grupos especialmente franceses hayan persistido en su
\end{abstract}

desarrollo y continuidad en épocas difíciles para su mantenimiento cuando la mayoría de los grupos que habían empezado la habían abandonado.

Afortunadamente las cosas han cambiado. En los últimos años, en la literatura médica se han aportado un número significativo de técnicas urológicas laparoscópicas con grandes series de casos que justifican su realización tanto desde el punto de vista técnico como de resultados en relación a la cirugía abierta. En el Congreso americano del 2004, se valoró la cirugía laparoscópìca como el patrón de oro en la cirugía renal.

En nuestro país, salvo excepciones, la mayoría de los grupos que han comenzado con la laparoscopia están en el inicio o en plena curva de aprendizaje. Es cierto que actualmente hay una fase de sensibilización muy positiva para su inicio y desarrollo que probablemente permita su instauración definitiva. Hay un interés enorme y una gran mayoría de grupos quieren introducirla en sus servicios. Para ello la primera reflexión para su verdadero desarrollo que se nos ocurre es que su docencia debe estar incluida de manera pormenorizada en el programa de los residentes. Por otro lado, al no tener laparoscopistas en la mayoría de los servicios su aprendizaje actual es una cuestión dificil y no podemos, por ahora, hacer un copilotaje con un laparoscopista experimentado al lado que es lo que da mayor garantía de formación. Sencillamente porque a día de hoy no los tenemos en la mayoría de los hospitales. Y la pregunta es ¿Qué hacer? ¿Cómo empezar? ¿A dónde dirigirnos? La solución no es fácil. Por todo ello nos decidimos a exponer los pasos que hemos dado en nuestro servicio para su desarrollo. Inicialmente pensamos en una estancia en un centro que se haga de forma rutinaria y pasar allí un "tiempo" de observador mirando como la realizan. Afortunadamente la laparoscopia se realiza con monitores que podemos seguir desde el quirófano o 
a través de un circuito cerrado de televisión. Es evidente que tras esta toma de contacto de 2 semanas o un mes no estamos capacitados para iniciar un programa similar en nuestro hospital pero nos ha estimulado para su inicio y en un futuro desarrollar un programa similar en nuestro hospital. La otra opción que puede ser alternativa o complementaria a la anterior es realizar un curso de cirugía experimental. Estos habitualmente duran de 2 a 3 dias y son interesantes ya que conseguimos realizar nuestras primeras técnicas de laparoscopia. Pero este curso no puede, a nuestro juicio, por si solo o con la estancia hospitalaria previa, a iniciar un programa clínico en nuestro centro. Se nos antoja complicado llegar a nuestro hospital y comenzar de esta manera un programa clínico de cirugía laparoscópica con buenos resultados, estable y de futuro.

Al problema ya se le ha encontrado una "salida" en otras especialidades, especialmente en cirugía general. La solución pasa por oficializar su aprendizaje.

Sería necesario un Diplomatura, un master? Depende del número de horas que queramos invertir en un curso de este tipo. Probablemente si. Porque se trata de una parte de nuestra especialidad que cada vez va adquirir mas protagonismo y en estos momentos no hay número suficiente de laparoscopistas en urología en el país para una formación dentro de la especialidad por vía MIR. Por ello creemos que hay que cambiar los planteamientos en su aprendizaje y tomar nota de otras experiencias en otras especialidades.

Las soluciones vendrán dadas en función del empeño que pongamos los urólogos de forma individual y en la facilitación que tengamos por parte de los centros hospitalarios

En el aprendizaje tenemos que asumir un "coste" elevado en tiempo y trabajo. Es necesario aceptarlo antes de comenzar. Está demostrado que el tener una formación sólida en cirugía abierta disminuye la conversión de cirugía laparoscópica a cirugía abierta. Pero, aún así, es necesario un periodo de tiempo en la sala de experimentación unida a una visita al quirófano en un hospital donde se haga laparoscopia urológica y el comienzo de un programa clínico con la ayuda de un experto en su inicio.
En nuestra opinión la formación debería comenzar por un curso de laparoscopia experimental intensivo en una unidad de cirugía experimental. En esta tiempo comenzaríamos a conocer el aparataje (monitor, insuflador, cámara, ...) , los sistemas de acceso a cavidad y el instrumental especializado (pinzas, tijeras, sistemas de irrigación y aspiración, aplicadores de clips, suturas, portaagujas, bisturís,etc ). El trabajo en los simuladores con visión directa, y con cámara, los ejercicios de prensión y de precisión de estructuras, etc. Tras esta primera fase realizaríamos sobre pletinas corte y suturas de modelos de dibujos que ya están bastante estandarizados y realizar también ejercicios sobre estructuras orgánicas. Nosotros hemos utilizado el estómago del cerdo y de la vaca. Debemos realizarlos con gran frecuencia hasta poder dominar la sutura de puntos sueltos o continúa con facilidad lo cual es "dificil". Tras esta fase pasaremos al animal. En la mayoría de los centros se utilizará el cerdo. Las razones son obvias; por su fácil obtención, por su precio, ya que no es muy caro obtenerlos y por sus estructuras anatómicas muy similares a las del hombre. Aquí se pueden estudiar las vías de abordaje, colocación de trócares, la exposición, la disección y la sutura. La nefrectomía es una intervención excelente para comenzar.

Una vez que hemos tomado "contacto" con la laparoscopia proponemos que se siga en el proceso de aprendizaje y desarrollo con un número de sesiones experimentales hasta completar 15 a 20 sesiones entendiendo por una sesión una mañana o una tarde completa. Esta programación se asociaría a asistencia a cirugía programada laparoscópica en un centro cercano al experimental. Esto se podría realizar en las diferentes comunidades. Se harian programaciones en algún centro de cirugía experimental consolidado y se formarían grupos de los hospitales interesados para poder desarrollar estos estudios en una especie de diplomatura de urología laparoscópica que ya existen en otras especialidades. Este programa se realizaría, al menos, por la dificultad de congregar a los profesionales en los cursos, como minimo en seis meses y en el se daría cuenta al final del número de técnicas e intervenciones que se hayan realizado. Una vez realizada esta fase comenzaríamos la fase clínica en cada centro de 
los urólogos que hayan realizado el curso para el inicio en su hospital acompañados por un laparoscopista con más experiencia al menos durante una semana en el centro donde se va iniciar el programa.

Es importante antes de comenzar establecer concretamente el tiempo que vamos a estar en cada fase y plantear las fechas para cada tiempo de aprendizaje. Incluso plantear la fecha de la primera laparoscopia en nuestro centro. Es decir realizar un riguroso calendario.

¿Cuántos deben empezar en cada centro? Nosotros creemos que dos. Creemos que la clave del éxito es no encontrarse solo en las primeras intervenciones y constituir un equipo de dos cirujanos muy motivados. La experiencia demuestra que cuando uno esta sólo es difícil conservar esa motivación lo que puede producir un número de abandonos considerable.

Es necesario escoger las intervenciones simples al principio que nos den un margen de confianza para tener éxito en las primeras intervenciones. Es importante tener días fijos a la semana para realizarla y no dejar que se haga una intervención laparoscópica de forma anecdótica ya que el programa terminaría por desaparecer.

La inclusión de nuevos miembros al equipo, como nos dijo el Doctor Bollens al cual estamos muy agradecidos ya que el fue nuestro "experto" debe ser unitaria, es decir, no incluir varios miembros a la vez, y obviamente una vez terminada la fase de aprendizaje de los que se han iniciado.
Probablemente dentro de unos años podamos manejar el aprendizaje de la laparoscopia como se hace con la cirugía abierta con un copilotaje con un laparoscopista experimentado que es lo que da mayor garantía de formación. Actualmente aún no es posible, porque a día de hoy aun no los tenemos en la mayoría de los hospitales. Por todo ello es imperativo en nuestro país iniciar la fase de aprendizaje primero en la sala de experimentación animal para seguir con un programa clínico de cirugía laparoscópica.

Creemos que hay muchos urólogos y residentes en nuestro país motivados para hacer el gran cambio que todo esto supone. Solo es cuestión de facilitar los medios para su inicio. Estos cambios originarán otros que ya se van vislumbrando como son los modelos informáticos para el aprendizaje, la miniaturización y mejoría del aparataje y la utilización de la cirugía robótica.

También creemos que existe un peligro claro de que si no nos enganchamos a estos cambios otras especialidades con tradición laparoscópica terminarán realizando las intervenciones de nuestra especialidad, cosa que ya sucede en algunos centros de nuestro país.

Dr. S. Novas Castro

C/ Río Dubra, 14. Bembibre.

15873 Valle del Dubra (La Coruña)

(Trabajo recibido el 26 enero de 2005) 